\title{
準静的成分と共振成分の連成を考慮した高層建築物の風応答評価法
}

\section{A New Model for Estimating Wind-induced Responses of Tall Buildings with Consideration of Cross-term between Background and Resonant Components}

\author{
王 天元 ${ }^{* 1}$ 植松 康 $^{* 2}$
}

Tianyuan WANG, Yasushi UEMATSU

\begin{abstract}
SUMMARY
In current design practice, the responses of buildings and structures to fluctuating wind loads are usually separated into the background and resonant components, based on the assumption that these two components are not correlated with each other. The present paper proposes a new model for estimating the wind-induced responses of tall buildings, in which the background and resonant components and their cross-term are strictly defined and included. Therefore, the new model is more exact than the conventional ones. Comparisons are made for the along-wind and across-wind responses of tall buildings between this model and the conventional ones, based on a parametric investigation. The results show that the conventional models give results similar to those obtained from the new model for the along-wind responses, but generally underestimate the across-wind responses. Finally, some conclusions and useful suggestions are presented.
\end{abstract}

Key words: $\quad$ Wind-induced response, Tall building, Background component, Resonant component, Cross-term

\section{Introduction}

At the beginning of the 1960s, Davenport ${ }^{1)-3)}$ developed a basic framework of modern wind engineering, in which the random process theory was introduced into wind-induced responses and the resultant load estimation of structures with consideration of the structural characteristics. In his early papers, only the fundamental mode of vibration was conveniently considered for computing the responses. Based on the displacement response spectrum of structure under fluctuating wind loads, the total response is represented by the sum of the quasi-static and resonant components; the quasi-static component is regarded as the integral of the response spectrum up to a 'high cut-off frequency' lower than the fundamental natural frequency of the structure. As a direct consequence, the total wind-induced response of the structure is based only on the fundamental generalized displacement.

Studies in the 1970s are divided into two approaches (Repetto and Solari $\left.{ }^{4}\right)$. The first followed the expression proposed by Davenport ${ }^{1-3)}$ in the 1960s. The second was based on the maximum load effect computed by using the influence line (or coefficients) for the response under consideration (e.g. base moment or shear force) in Europe (e.g. ESDU $1976^{5}$ ).

In the 1980s, Solari' ${ }^{6}$ derived a closed-form solution for the along-wind responses, in which he first called the quasi-static component as 'Background Component'. Kasperski ${ }^{7)}$ proposed the 'Load Response Correlation (LRC) method', which is

* 1 東北大学大学院工学研究科都市 - 建築学専攻 大学院生

Graduate Student, Department of Architecture and Building Science, Graduate School of Engineering, Tohoku University * 2 東北大学大学院工学研究科都市・建築学専攻 教授

Professor, Department of Architecture and Building Science, Graduate School of Engineering, Tohoku University (原稿受理年月日：2010 年 4 月 7 日，採用決定：2010 年 8 月 3 日） 
useful to determine the distribution of quasi-static wind loads for the load effect under consideration.

The dynamic responses of MDOF structures with distributed mass can be computed by using the modal analysis. However, this method is not efficient for tall buildings, as Holmes ${ }^{8)}$ pointed out. Higher mode responses of tall buildings under fluctuating wind loads are usually quasi-static. If we consider only the fundamental mode for estimating the quasi-static response, the response may be underestimated. Hence, Davenport ${ }^{9), 10)}$ proposed a new framework for evaluating the total response in the 1990s; this framework is called 'Original definition' in the present paper. In this framework, the total equivalent static response is separated into the background and resonant components. The background component is defined as the response in a frequency range lower than the fundamental natural frequency and determined by using the influence line function. The resonant component is determined from the inertial response in a frequency range near the fundamental natural frequency. Following the 'Original definition', several researchers (e.g. Holmes ${ }^{11)}$, Kareem and Zhou ${ }^{12)}$, Repetto and Solari ${ }^{4)}$, Chen and Kareem ${ }^{13)}$ ) have developed some strict and practical models or frameworks for evaluating the load effect. However, if the 'Original definition' is investigated carefully, it may be found that the 'Original definition' of the background and resonant components is misleading in a strict sense. The cut-off frequency representing the boundary of these two components is not clearly defined.

This paper presents a new model for estimating the wind-induced responses of tall buildings, in which the background and resonant components and their cross-term are formulated for the sake of load effects. These terms are strictly defined and have clear physical meanings. Therefore, the new model is more exact than the conventional ones. Comparisons are made for the along-wind and across-wind responses between this new model and the conventional ones, based on a parametric investigation. The results show that the conventional models underestimate the across-wind responses. Finally, the theoretical and practical meanings of this model are discussed and some conclusions and useful suggestions are presented.

\section{Starting point of the 'Original definition'}

The equation of motion for an $N$-story building may be given by the following equation:

$$
[M]\{\ddot{u}(t)\}+[C]\{\dot{u}(t)\}+[K]\{u(t)\}=\{p(t)\}
$$

where $[M],[C]$ and $[K]$ are respectively the mass, damping and stiffness matrices of the building; $\{\ddot{u}(t)\},\{\dot{u}(t)\}$ and $\{u(t)\}$ are respectively the acceleration, velocity and displacement response vectors; and $\{p(t)\}$ is the vector of external fluctuating wind loads.

Huang and Chen ${ }^{14)}$ indicated that the resonant effects of higher modes on the load effects at the base are negligibly small. Therefore, in the analysis of the equivalent static responses, or load effects for a tall building, only the fundamental mode is considered. In this case, $\{\ddot{u}(t)\},\{\dot{u}(t)\}$ and $\{u(t)\}$ are respectively represented by the product of the fundamental generalized acceleration $\ddot{\xi}_{1}(t)$, velocity $\dot{\xi}_{1}(t)$, displacement $\xi_{1}(t)$ and mode shape $\left\{\phi_{1}\right\}$ as follows:

$\{\ddot{u}(t)\} \approx \ddot{\xi}_{1}(t)\left\{\phi_{1}\right\},\{\dot{u}(t)\} \approx \dot{\xi}_{1}(t)\left\{\phi_{1}\right\},\{u(t)\} \approx \xi_{1}(t)\left\{\phi_{1}\right\}(2)$

Substituting Eq. (2) into Eq. (1) and considering the orthogonality of mode shapes, we obtain the following equation:

$$
m_{1}^{*} \ddot{\xi}_{1}(t)+c_{1}^{*} \dot{\xi}_{1}(t)+k_{1}^{*} \xi_{1}(t)=P_{1}^{*}(t)
$$

where $m_{1}^{*}$ is the generalized mass, and $m_{1}^{*}=\left\{\phi_{1}\right\}^{T}[M]\left\{\phi_{1}\right\} ; c_{1}^{*}$ is the generalized damping, and $c_{1}^{*}=4 \pi f_{1} \zeta_{1} m_{1}^{*}$ in which $f_{1}$ and $\zeta_{1}$ are the fundamental natural frequency and critical damping ratio; $k_{1}^{*}$ is the generalized stiffness, and $k_{1}^{*}=\left(2 \pi f_{1}\right)^{2} m_{1}^{*}$; and $P_{1}^{*}(t)$ is the generalized fluctuating wind force, and $P_{1}^{*}(t)=\left\{\phi_{1}\right\}^{T}\{p(t)\}$.

Because $\{p(t)\}$ in Eq. (1) is based on the steady state situation, it may be given by the Fourier series as follows:

$$
\{p(t)\}=\sum_{f}\left\{p_{f}\right\} e^{i 2 \pi f t}, \quad(i=\sqrt{-1})
$$

Furthermore, $\xi_{1}(t)$ may also be expressed by

$$
\xi_{1}(t)=\sum_{f} \xi_{1 f} e^{i 2 \pi f t}
$$

where $f$ is frequency; $\left\{p_{f}\right\}$ and $\xi_{1 f}$ are the amplitude functions in the frequency domain corresponding to $\{p(t)\}$ and $\xi_{1}(t)$ in the time domain, respectively. Substituting Eqs. (4) and (5) into Eq. (3), we may obtain the following equation:

$$
\sum_{f}\left\langle 1-\left(f / f_{1}\right)^{2}+2 i \zeta_{1}\left(f / f_{1}\right)\right\rangle \xi_{1 f} e^{i 2 \pi f t}=\sum_{f} P_{1 f}^{*} e^{i 2 \pi f t} / k_{1}^{*}(6)
$$

where $P_{1 f}^{*}=\left\{\phi_{1}\right\}^{T}\left\{p_{f}\right\}$. Because of the stationary characteristic of external wind loads, Eq. (6) may be solved in a certain frequency. The fundamental displacement $\xi_{1 f}$ in the frequency domain may be given by

$$
\begin{gathered}
\xi_{1 f}=H_{1}(f) \frac{P_{1 f}^{*}}{k_{1}^{*}} \\
H_{1}(f)=\frac{1}{1-\left(f / f_{1}\right)^{2}+2 i \zeta_{1}\left(f / f_{1}\right)}
\end{gathered}
$$


To get the response to the external wind force with consideration of the structural characteristics of the building, the 'Direct method' was adopted by Davenport ${ }^{1)-3)}$. In this method, the equivalent static response, or load effect may be given by

$$
r(t)=\{I\}^{T}[K]\{u(t)\} \approx \xi_{1}(t)\{I\}^{T}[K]\left\{\phi_{1}\right\}
$$

where $\{I\}$ is the influence coefficient vector that depends on the response under consideration. The superscript ' $T$ ' stands for transposing of vector. The 'Direct method' is widely used in the field of earthquake engineering, because the resonant component is dominant in the response and it is easy to get the response spectrum by using this method. The analysis framework proposed by Davenport ${ }^{1-3)}$ in the 1960s might be significantly influenced by this method. Considering the fundamental mode shape vector as the influence coefficient vector and substituting $\{I\}^{T}=\left\{\phi_{1}\right\}^{T}$ into Eq. (9), the fundamental generalized force with consideration of the structural characteristics may be obtained, as was done by Davenport in the 1960s.

Later, the other researchers (e.g. Vickery ${ }^{15)}$ ) found that considering the contribution of only the fundamental mode to the background component was not enough to predict the response accurately. They proposed new methods for evaluating the background component by using the influence line on the basis of the quasi-static assumption. But they have yet followed the 'Original definition' of the Davenport's framework.

The 'Original definition' is based on the assumption that the background and resonant components are not correlated with each other. If the peak of the background component is far away from that of the resonant component in the response spectrum, as shown in Fig. 1, this ‘Original definition' may be reasonable.

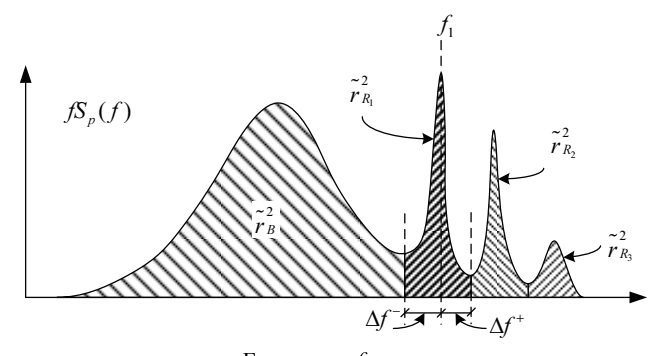

Frequency $f$

Fig.1 Background and resonant components of response spectrum ${ }^{10)}$

Based on the 'Original definition', the standard deviation $\sigma_{B O}$ of the background component may be described as follows $\left(\right.$ Chen and Kareem $^{13)}$ ):

$$
\sigma_{B O}=\left[\int_{0}^{f_{1}-\Delta f^{-}} S_{P_{r}^{*}}(f) d f\right]^{1 / 2}
$$

where $S_{P_{r}^{*}}(f)$ is the wind load spectrum based on the generalized force $P_{r}^{*}(t)$ with consideration of load effect (e.g. base moment or shear), in which the influence line $I(z)$, with $z$ being the coordinate in the vertical direction, is considered (also see Eq. (22) ). $\Delta f^{-}$defines the upper limit of integral; it is some value ranging from 0 to $f_{1} / 2$ for the along-wind responses ${ }^{16)}$. The standard deviation $\sigma_{R O}$ of the resonant component may be given by

$$
\begin{gathered}
\sigma_{R O}=\left(2 \pi f_{1}\right)^{2} \sigma_{R, \xi_{1}} \int_{0}^{H} m(z) I(z) \phi_{1}(z) d z \\
\sigma_{R, \xi_{1}}=\frac{1}{k_{1}^{*}}\left[\int_{f_{1}-\Delta f^{-}}^{f_{1}+\Delta f^{+}}\left|H_{1}(f)\right|^{2} S_{P_{1}^{*}}(f) d f\right]^{1 / 2}=\frac{1}{k_{1}^{*}} \sqrt{\frac{\pi}{4 \zeta_{1}} f_{1} S_{P_{1}^{*}}\left(f_{1}\right)}
\end{gathered}
$$

where $m(z)$ represents the distributed mass in the vertical direction; $\sigma_{R, \xi_{1}}$ is the standard deviation of the fundamental generalized resonant displacement; $S_{P_{1}^{*}}(f)$ is the power spectrum of the fundamental generalized wind force. Furthermore, the resonant component can be computed by using the 'White-noise Approximation' technique ${ }^{17)}$.

Thus, the standard deviation of the total equivalent static load may be given by the following equation:

$$
\sigma_{\text {Ori. }}=\sqrt{\sigma_{B O}^{2}+\sigma_{R O}^{2}}
$$

\section{New model}

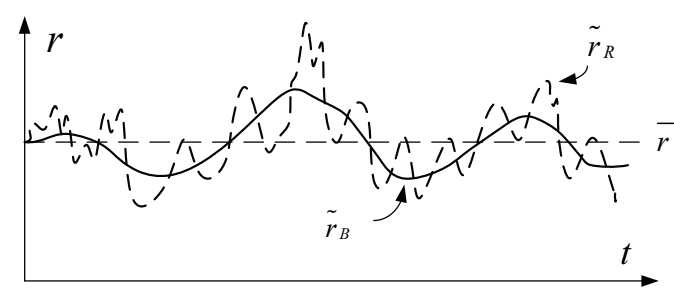

Fig.2 Background and resonant components in time history response ${ }^{10)}$

Fig.2 shows a schematic illustration of the time history of the background and resonant components. The total fluctuating response $r(t)$ may be expressed as follows:

$$
r(t)=\tilde{r}_{B}(t)+\tilde{r}_{R}(t)
$$

where $\tilde{r}_{B}(t)$ and $\tilde{r}_{R}(t)$ respectively represent the background and resonant components. When Eq. (14) is translated into the expression of spectrum response in the frequency domain, the following equation can be obtained:

$$
\sigma_{r}^{2}=\sigma_{B}^{2}+\sigma_{R}^{2}+2 \rho_{B R} \sigma_{B} \sigma_{R}
$$

where $2 \rho_{B R} \sigma_{B} \sigma_{R}$ represents the cross-term between the 
background and resonant components, which is usually neglected in the conventional models; and $\rho_{B R}$ represents the correlation coefficient between $\tilde{r}_{B}(t)$ and $\tilde{r}_{R}(t)$. Actually, the cross-term always exists in a strict sense. Thus, the expression of Eq. (15) is regarded as an exact expression for the total fluctuating response.

In the following, a new model is derived. The 'Original definition' is obtained directly from Eq. (1), as was discussed in Sec. 2. On the other hand, the equivalent static response may be indirectly obtained from the sum of the external wind load term, the inertial force term and the damping force term in Eq. (1). This method is called the 'Indirect method' in the present paper.

In the 'Indirect method', we obtain the following equation from Eq. (1):

$$
[K]\{u(t)\}=\{p(t)\}-[M]\{\ddot{u}(t)\}-[C]\{\dot{u}(t)\}
$$

where the damping matrix $[C]$ may be represented by the following equation, based on the superposition of Modal Damping Matrix method $\left(\right.$ Chopra $\left.^{18)}\right)$ :

$$
[C]=\frac{2 \zeta_{1} \cdot\left(2 \pi f_{1}\right)}{m_{1}^{*}}[M]\left\{\phi_{1}\right\}\left\{\phi_{1}\right\}^{T}[M]
$$

Multiplying the both sides of Eq. (16) by $\left\{I^{T}\right.$, we obtain the load effect $r(t)$ as follows:

$$
r(t)=\{I\}^{T}\{p(t)\}-\{I\}^{T}\langle[M]\{\ddot{u}(t)\}+[C]\{\dot{u}(t)\}\rangle
$$

Now, let us consider the vertical mass distribution $m(z)$, the influence line $I(z)$ and the fundamental mode shape $\phi_{1}(z)$, which are expressed by the following equations:

$$
m(z)=m_{0}\left(1-\lambda \frac{z}{H}\right)
$$

where $m_{0}$ is the mass per unit height at the bottom of the building, $H$ is the height of roof top of building; and $\lambda$ is a constant $(0 \leq \lambda \leq 1)$.

$$
I(z)=i_{0}(z / H)^{\beta_{0}}
$$

where the values of $\beta_{0}$ and $i_{0}$ depend on the load effect under consideration; for example, $\beta_{0}=1$ and $i_{0}=H$ for the base moment, and $\beta_{0}=0$ and $i_{0}=1$ for the base shear force.

$$
\phi_{1}(z)=(z / H)^{\beta}
$$

where $\beta$ is an exponent of the fundamental mode shape ranging from 0.5 to 1.5 for typical buildings.

In the present study, only the fundamental mode is considered in the new model in the same manner as that was done in the 'Original definition' model. We obtain the following equation from Eqs. (17) and (18):

$$
r(t) \approx r_{\text {New }}(t)=P_{r}^{*}(t)-m_{r 1}^{*}\left\langle\ddot{\xi}_{1}(t)+2 \zeta_{1} \cdot 2 \pi f_{1} \cdot \dot{\xi}_{1}(t)\right\rangle(22)
$$

where $P_{r}^{*}(t)=\int_{0}^{H} p(z, t) I(z) d z$, in which $p(z, t)$ is the external wind load at height $z$; and $m_{r 1}^{*}=\int_{0}^{H} m(z) I(z) \phi_{1}(z) d z$.

Substituting Eqs. (5), (7) and (8) into Eq. (22), we obtain the following equation:

$$
r_{\text {New }}(t)=P_{r}^{*}(t)+\sum_{f} \frac{m_{r 1}^{*}}{m_{1}^{*}}\left\langle H_{1}(f)-1\right\rangle P_{1 f}^{*} e^{i 2 \pi f t}
$$

where $P_{r}^{*}(t)=\sum_{f} P_{r f}^{*} e^{i 2 \pi f t}$ represented by the Fourier series. Furthermore we can obtain the following equation:

$$
r_{\text {New }}(t)=\sum_{f}\left\{P_{r f}^{*}+\frac{m_{r 1}^{*}}{m_{1}^{*}}\left\langle H_{1}(f)-1\right\rangle P_{1 f}^{*}\right\} e^{i 2 \pi f t}
$$

where the generalized mass $m_{1}^{*}=\int_{0}^{H} m(z) \phi_{1}(z) \phi_{1}(z) d z$. We also find that $P_{1}^{*}(t)=\int_{0}^{H} p(z, t) \phi_{1}(z) d z=\sum_{f} P_{1 f}^{*} e^{i 2 \pi f t}$.

Eq. (23) is similar to Eq. (14). The first term of the right hand side is directly obtained from the wind load term in Eq. (1), treated as the background component, and the second term from the inertial and damping force terms, treated as the resonant component in the present paper.

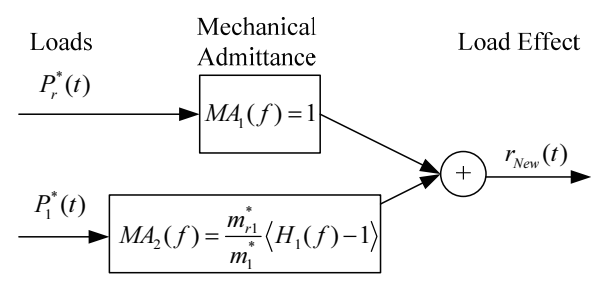

Fig.3. Two inputs and one output system ${ }^{19)}$

Applying the random vibration theory (Newland ${ }^{19)}$ ) of two inputs and one output system to Eq. (24), as shown in Fig. 3, the response spectrum of this system may be represented by

$$
\begin{aligned}
S_{r}(f)= & M A_{1}(f) M A_{1}^{\prime}(f) S_{P_{r}^{*}}(f)+M A_{1}(f) M A_{2}^{\prime}(f) S_{P_{r}^{*} P_{1}^{*}}(f) \\
& +M A_{1}^{\prime}(f) M A_{2}(f) S_{P_{1}^{*} P_{r}^{*}}(f)+M A_{2}(f) M A_{2}^{\prime}(f) S_{P_{1}^{*}}(f)
\end{aligned}
$$

where $M A_{i}(f)(i=1,2)$ is the mechanical admittance function; the prime " "' stands for the complex conjugate, e.g., $M A_{1}(f)$ is the complex conjugate of $M A_{1}(f)$. From Eq. (25), the standard deviations, $\sigma_{B N}$ and $\sigma_{R N}$, of the background and resonant components in the new model may be expressed as follows:

$$
\sigma_{B N}=\left[\int_{0}^{+\infty} S_{P_{r}^{*}}(f) d f\right]^{1 / 2}
$$




$$
\sigma_{R N}=\frac{m_{r 1}^{*}}{m_{1}^{*}}\left[\int_{0}^{+\infty}\left|H_{1}(f)-1\right|^{2} S_{P_{1}^{*}}(f) d f\right]^{1 / 2}
$$

The cross-term in the present model may be represented by

$$
2 \rho_{B R} \sigma_{B N} \sigma_{R N}=\frac{m_{r 1}^{*}}{m_{1}^{*}} \int_{0}^{+\infty}\left\langle H_{1}(f)+H_{1}^{\prime}(f)-2\right\rangle S_{P_{r}^{*} P_{1}^{*}}(f) d f
$$

Substituting Eqs. (26) and (27) into Eq. (28), the correlation coefficient $\rho_{B R}$ between the background and resonant components may be given by

$$
\rho_{B R}=\frac{1}{2} \frac{\int_{0}^{+\infty}\left\langle H_{1}(f)+H_{1}^{\prime}(f)-2\right\rangle S_{P_{r}^{*} P_{i}^{*}}(f) d f}{\left[\int_{0}^{+\infty} S_{P_{r}^{*}}(f) d f\right]^{1 / 2}\left[\int_{0}^{+\infty}\left|H_{1}(f)-1\right|^{2} S_{P_{1}^{*}}(f) d f\right]^{1 / 2}}
$$

The wind load spectrum $S_{P_{r}^{*}}(f)$ in Eq. (26) with consideration of the influence line $I(z)$ may be given by

$$
S_{P_{r}^{\prime}}(f)=\int_{0}^{H} \int_{0}^{H} I\left(z_{1}\right) I\left(z_{2}\right) S_{p}\left(z_{1}, z_{2}, f\right) d z_{1} d z_{2}
$$

The cross-spectrum in Eq. (28) between the influence line $I(z)$ and the fundamental mode shape $\phi_{1}(z)$ may be given by

$$
S_{P_{r}^{*} P_{1}^{*}}(f)=\int_{0}^{H} \int_{0}^{H} I\left(z_{1}\right) \phi_{1}\left(z_{2}\right) S_{p}\left(z_{1}, z_{2}, f\right) d z_{1} d z_{2}
$$

And the power spectrum of the fundamental generalized wind force may be given by

$$
S_{P_{1}^{*}}(f)=\int_{0}^{H} \int_{0}^{H} \phi_{1}\left(z_{1}\right) \phi_{1}\left(z_{2}\right) S_{p}\left(z_{1}, z_{2}, f\right) d z_{1} d z_{2}
$$

where $S_{p}\left(z_{1}, z_{2}, f\right)$ is the cross-spectrum between $p\left(z_{1}, t\right)$ and $p\left(z_{2}, t\right)$. Furthermore, we can get the flowing expression of the new model for the load effect, which is the same to Eq. (15):

$$
\sigma_{\text {New }}=\sqrt{\sigma_{B N}^{2}+\sigma_{R N}^{2}+2 \rho_{B R} \sigma_{B N} \sigma_{R N}}
$$

In the process of derivation, the 'Original definition' is derived from the 'Direct method', while the new model is from the 'Indirect method'. The 'Original definition' is useful, but it may be a vague definition in a strict sense. The new model with a strict definition of the background and resonant components and their cross-term gives a more exact estimation of the responses, but it is complicated because of the existence of the cross-term. The new model can be applied to the data processing of wind tunnel experiments for evaluating the responses more accurately.

The 'Original definition' model is essentially based on the SRSS (Square Root of the Sum of the Squares) method, while the new model is essentially based on the CQC (Complete Quadratic Combination) method. It is thought that the CQC method provides more accurate estimation of the responses than the SRSS method.

\section{Comparison for the along-wind responses between the new model and the 'Original definition' models}

Regarding the along-wind responses of tall buildings, we focus on two limiting situations (i.e. low correlation and high correlation levels) because of the complexity of aerodynamic admittance function ( $\mathrm{Xu}$ and $\mathrm{Kwok}^{20)}$ ).

The corss-spectrum in Eqs. (30) to (32) may be expressed as follows:

$$
S_{p}\left(z_{1}, z_{2}, f\right)=W\left(z_{1}\right) W\left(z_{2}\right) S_{u}^{*}(f) R\left(z_{1}-z_{2}, f\right)
$$

where $W(z)=W_{H}(z / H)^{\alpha}$ is the amplitude of fluctuating wind load at height $z ; \alpha$ is the power law exponent of the mean wind velocity profile; $R\left(z_{1}-z_{2}, f\right)$ is the cross-correlation function between the fluctuating wind loads at heights $z_{1}$ and $z_{2}$; and $S_{u}^{*}(f)$ is the normalized power spectrum of wind velocity fluctuations. In the present paper, von Karman spectrum, represented by the following equation, is adopted on referring to the AIJ Recommendations for Loads on Buildings ${ }^{21)}$.

$$
S_{u}^{*}(f)=\frac{4 \frac{1}{f_{1} V_{a l}}}{\left[1+71\left(\frac{f}{f_{1}} \frac{1}{V_{a l}}\right)^{2}\right]^{5 / 6}}
$$

where $V_{a l}=U_{H} /\left(f_{1} L_{H}\right)$ is a nondimensional parameter; $f_{1}$ is the fundamental natural frequency of the building; $U_{H}$ and $L_{H}$ are the mean wind velocity and the turbulence integral scale at the level of roof top $H$, respectively.

For the two limiting situations, the formulas of $R\left(z_{1}-z_{2}, f\right)$ and others may be given as follows:

1. In the case of low correlation level

For the low correlation level, the cross-correlation function can be assumed as

$$
R\left(z_{1}-z_{2}, f\right)=\left\{\begin{array}{lll}
1 & \text { for } & z_{1}=z_{2} \\
0 & \text { for } & z_{1} \neq z_{2}
\end{array}\right.
$$

Substituting Eqs. (34) and (36) into Eqs. (30) to (32), we get the following equations:

$$
\begin{aligned}
S_{P_{r}^{*}}(f) & =\frac{i_{0}^{2} H}{1+2 \alpha+2 \beta_{0}} W_{H}^{2} S_{u}^{*}(f) \\
S_{P_{r}^{*} P_{1}^{*}}(f) & =\frac{i_{0} H}{1+2 \alpha+\beta_{0}+\beta} W_{H}^{2} S_{u}^{*}(f) \\
S_{P_{1}^{*}}(f) & =\frac{H}{1+2 \alpha+2 \beta} W_{H}^{2} S_{u}^{*}(f)
\end{aligned}
$$

2. In the case of high correlation level 
Another limiting situation for the high correlation level can be assumed as

$$
R\left(z_{1}-z_{2}, f\right) \equiv 1
$$

In the same manner as was done for the low correlation level, the following equations may be obtained:

$$
S_{P_{r}^{*}}(f)=\left(\frac{i_{0} H}{1+\alpha+\beta_{0}}\right)^{2} W_{H}^{2} S_{u}^{*}(f)
$$

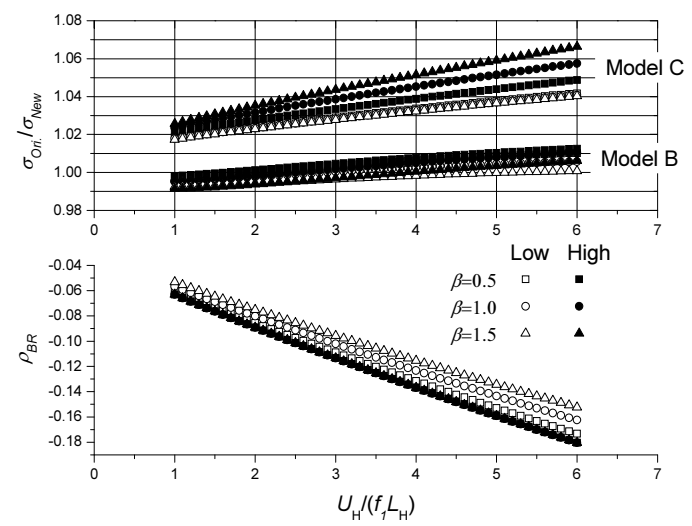

(a) $\alpha=0.15, \zeta_{1}=3 \%$

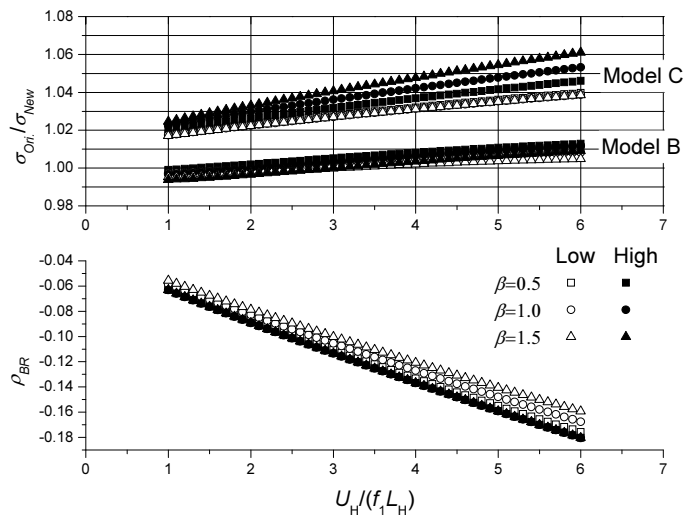

(b) $\alpha=0.35, \zeta_{1}=3 \%$
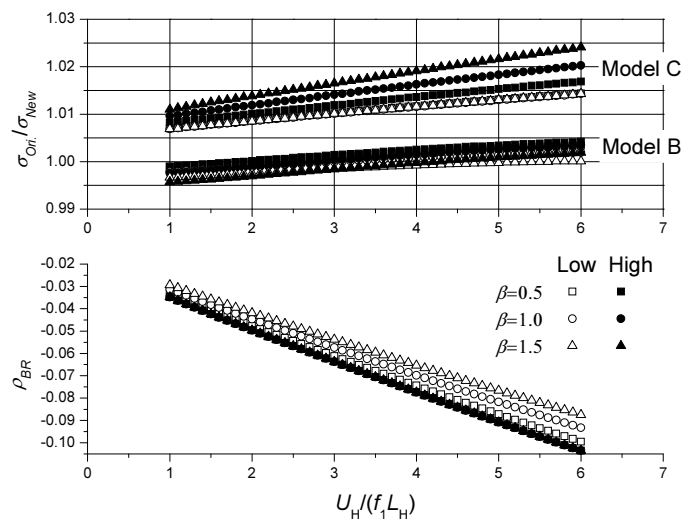

(c) $\alpha=0.15, \zeta_{1}=1 \%$

Fig. 4. The $\sigma_{\text {Ori. }} / \sigma_{\text {New }}$ ratio for Models $\mathrm{B}$ and $\mathrm{C}$ and the correlation coefficient $\rho_{B R}$ (Load effect: base shear)

$$
\begin{gathered}
S_{P_{r}^{*} P_{1}^{*}}(f)=\frac{i_{0} H^{2}}{\left(1+\alpha+\beta_{0}\right)(1+\alpha+\beta)} W_{H}^{2} S_{u}^{*}(f) \\
S_{P_{1}^{*}}(f)=\left(\frac{H}{1+\alpha+\beta}\right)^{2} W_{H}^{2} S_{u}^{*}(f)
\end{gathered}
$$

Comparisons are made for the standard deviation of the total response (load effect) between the following three models, A to C.

A: New model proposed in this paper.

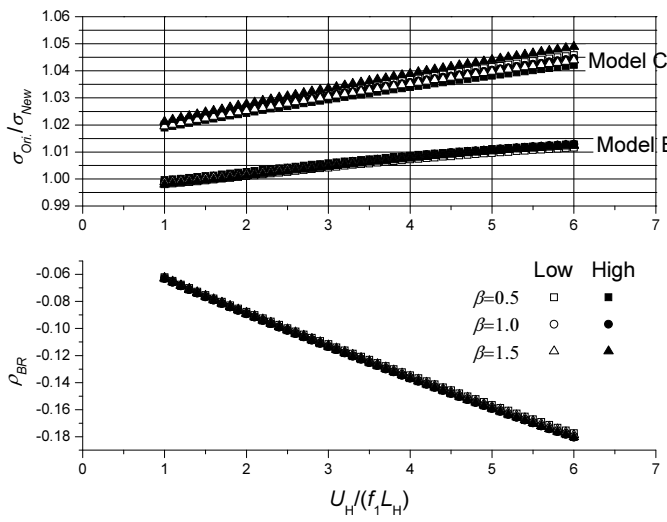

(a) $\alpha=0.15, \quad \zeta_{1}=3 \%$

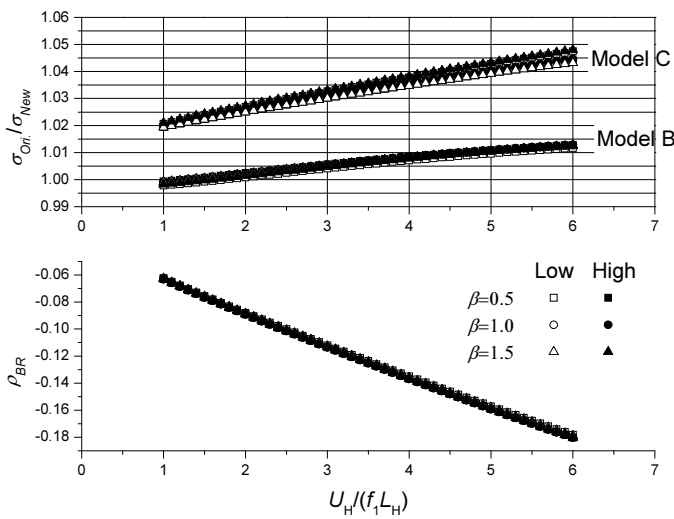

(b) $\alpha=0.35, \zeta_{1}=3 \%$
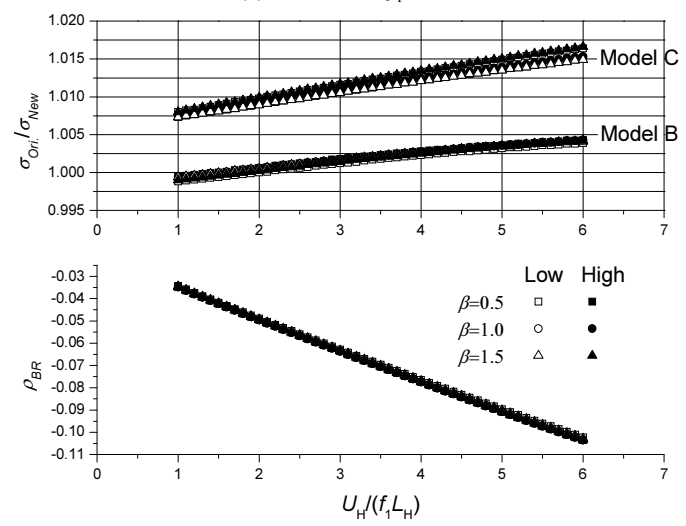

(c) $\alpha=0.35, \zeta_{1}=1 \%$

Fig. 5. The $\sigma_{O r i .} / \sigma_{N e w}$ ratio for Models B and $\mathrm{C}$ and the correlation coefficient $\rho_{B R}$ (Load effect: base moment) 
B: 'Original definition' model with $\Delta f^{-}=0$ in Eq. (10).

C: 'Original definition' model with $\Delta f^{-}=-\infty$ in Eq. (10); i.e. the integral over the whole frequency range.

Note that Model $\mathrm{C}$ is widely used in codes and standards. Assuming that the mass $m(z)$ is constant, i.e. $\lambda=0$ in Eq. (19), we obtain the following equation:

$$
\frac{m_{r 1}^{*}}{m_{1}^{*}}=\frac{i_{0}(2 \beta+1)}{\left(\beta+\beta_{0}+1\right)}
$$

The formulas of $\sigma_{O r i} / \sigma_{N e w}$ for the low and high correlation levels are presented in Appendix, where $\sigma_{\text {Ori. }}$ and $\sigma_{\text {New }}$ are respectively the standard deviations obtained from the 'Original definition' and the new models. From these formulas, it is found that the $\sigma_{\text {Ori. }} / \sigma_{\text {New }}$ ratio is a function of $V_{a l}, \zeta_{1}, \alpha, \beta_{0}$ and $\beta$ in the same manner as for $\rho_{B R}$ in Eq. (29). Indeed, the actual values of $\sigma_{O r i .} / \sigma_{N e w}$ and $\rho_{B R}$ exist between the values for these two limiting conditions, due to the aerodynamic admittance function.

In most cases, the nondimensional parameter $V_{a l}$ ranges from 1 to 6 . The exponent $\beta$ of mode shape ranges from 0.5 to 1.5. Here, three values of $\beta$, i.e. $\beta=0.5,1.0$ and 1.5 are used. The exponent $\alpha$ of the mean wind velocity profile is 0.15 for Terrain category II and 0.35 for Terrain category $\mathrm{V}$, based on the AIJ-RLB ${ }^{21)}$. The critical damping ratio of the buildings is assumed $1 \%$ or $3 \%$.

Figs. 4 and 5 show the $\sigma_{\text {Ori. }} / \sigma_{\text {New }}$ ratios for Models B and C together with the correlation coefficient $\rho_{B R}$; the load effects under consideration are the base shear force in Fig. 4 and the base moment in Fig. 5. From these figures we can observe the following features:

(1) Model B seems to provide accurate estimation of the load effects. The results for Model B agree well with those for Model A (the new model); the difference is less than approximately $1 \%$ within the limits of this analysis.

(2) Model C generally overestimates the load effects. In this model, the background component is regarded as a quasi-static response to the external fluctuating loads including entire frequency range, which results in somewhat larger estimation of the background component, as shown in Fig. 1. However, the difference in the standard deviation between Models $\mathrm{C}$ and $\mathrm{A}$ is no more than $5 \%$, which may be acceptable in practical applications.

(3) The correlation coefficient $\rho_{B R}$ computed from Model A is generally negative. If the cross-term in Model A is neglected, the results will be close to those from Model C.

\section{Comparison for the across-wind responses between the} new model and the 'Original definition' models

For calculating the across-wind responses of tall buildings (Width $B \times$ Depth $D \times$ Height $H$ ), the power spectrum $S_{M}(f)$ of the base moment $M(t)$ given in the AIJ-RLB ${ }^{21)}$ is adopted here. In the case where $B=D$, it is represented as follows:

$$
\frac{f S_{M}(f)}{\left(0.5 \rho U_{H}^{2} B H^{2}\right)^{2}}=\frac{S_{s}\left(\frac{f}{f_{1}} \frac{1}{V_{a c} f_{s}}\right)^{2}}{\left[1-\left(\frac{f}{f_{1}} \frac{1}{V_{a c} f_{s}}\right)^{2}\right]^{2}+4 \beta_{s}^{2}\left(\frac{f}{f_{1}} \frac{1}{V_{a c} f_{s}}\right)^{2}}
$$

where $V_{a c}=U_{H} /\left(f_{1} B\right)$ is a reduced wind velocity; $S_{s}$ is the amplitude parameter; $f_{s}$ is the peak frequency depending on $B$ and $D$; and $\beta_{s}$ is a constant related to the bandwidth, which also depends on $B$ and $D$.

Here, to obtain the cross-spectrum $S_{M P_{1}^{*}}(f)$ between $M(t)$ and $P_{1}^{*}(t)$ based on Eq. (31), the relationship between $S_{M P_{1}^{*}}(f)$ and $S_{M}(f) S_{P_{1}^{*}}(f)$ is investigated. In the same manner as for the along-wind responses, we consider the two limiting situations for the across-wind responses, where $\alpha$ in Eq. (34) is replaced by $2 \alpha^{20)}$. Therefore, for the low correlation level, the following equation may be obtained:

$$
\frac{S_{M P_{1}^{*}}(f)}{\sqrt{S_{M}(f) S_{P_{1}^{*}}(f)}}=\frac{\sqrt{3+4 \alpha} \sqrt{1+4 \alpha+2 \beta}}{2+4 \alpha+\beta}
$$

On the other hand, for the high correlation level, the following assumption can be made:

$$
\frac{S_{M P_{1}^{*}}(f)}{\sqrt{S_{M}(f) S_{P_{1}^{*}}(f)}} \equiv 1
$$

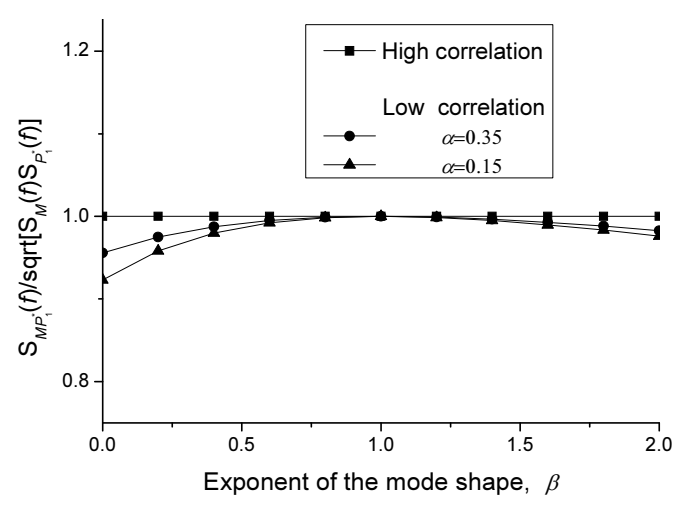

Fig.6. Variation of $S_{M P_{1}^{*}}(f) / \sqrt{S_{M}(f) S_{P_{1}^{\prime}}(f)}$ with $\beta$

Fig. 6 shows the variation of $S_{M P_{1}^{*}}(f) / \sqrt{S_{M}(f) S_{P_{1}^{*}}(f)}$ with the exponent $\beta$ of the mode shape for the low and high 
correlation levels. It is found that the ratios are close to 1.0 for a range of $\beta$ from 0.5 to 1.5 even for the low correlation level. Therefore, we can assume the perfect correlation between $M(t)$ and $P_{1}^{*}(t)$, i.e. $S_{M P_{1}^{*}}(f) / \sqrt{S_{M}(f) S_{P_{1}^{*}}(f)}=1$.

Generally, $S_{P_{1}^{*}}(f)$ is represented by $S_{M}(f)$ as follows ${ }^{20)}$ :

$$
S_{P_{1}^{*}}(f)=\frac{\eta^{2}}{H^{2}} S_{M}(f)
$$

where $\eta$ is the mode shape correction coefficient. Because $S_{M P_{1}^{*}}(f) / \sqrt{S_{M}(f) S_{P_{1}^{*}}(f)}=1$, as mentioned above, we obtain:

$$
S_{M P_{1}^{*}}(f)=\frac{\eta}{H} S_{M}(f)
$$

Now, comparisons are made for the standard deviation of the total response (load effect: base moment) between the 'Original definition' models (Models B and C) and the new model
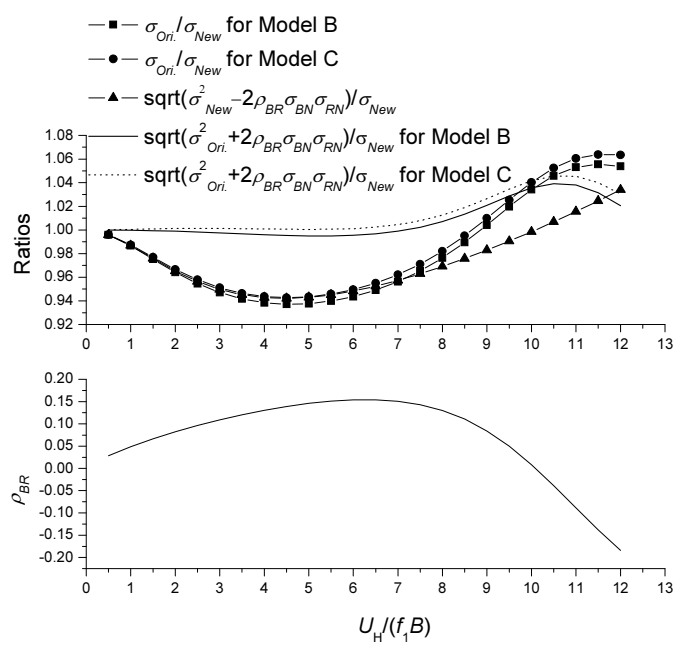

(a)

Fig.7. The $\sigma_{O r i .} / \sigma_{N e w}$ ratios for Models B and $\mathrm{C}$ and the correlation coefficient $\rho_{B R}$ (Load effect: base moment) (a) $\alpha=0.15, \zeta_{1}=3 \%, \beta=0.5$; (b) $\alpha=0.35, \zeta_{1}=1 \%, \beta=1.5$
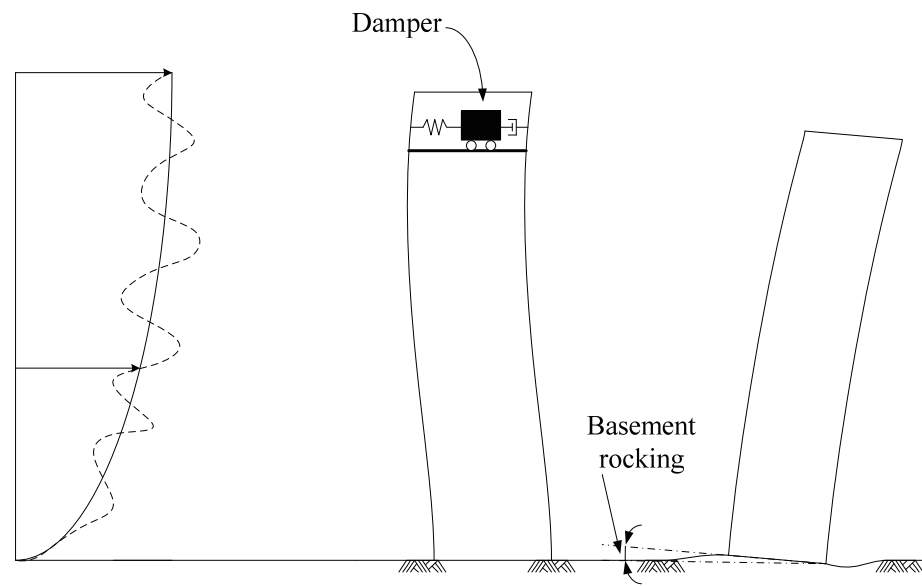

Isolators

Fig.8. Tall buildings with artificial dampers, with SSI effect and with seismic isolators 
'Original definition' models) are very close to each other. These results generally underestimate the responses. The error is larger than $5 \%$ when $V_{a c}$ is in a range from 2 to 8 , which may not be acceptable in practical applications. The lower the damping of tall buildings is, the smaller the relative error is.

(2) When the cross-term is neglected in the new model, the results are close to those obtained from the 'Original definition' models (Models B and C). Adding the cross-term effect to the results obtained from the 'Original definition' models can provide more accurate estimation of the total responses, as shown in Fig. 7 (solid and dotted lines in the figures).

(3) The correlation coefficient $\rho_{B R}$ for Model A is generally positive when $V_{a c}=U_{H} /\left(f_{1} B\right) \leq 10$, i.e. in a reduced wind velocity range of practical applications. This feature is related to a fact that the 'Original definition' models underestimate the total responses.

\section{Concluding remarks}

In the present paper, a new model including the background and resonant components and their cross-term is derived, for analyzing the wind-induced responses of tall buildings based on the 'Indirect method'.

In this model, the background component is essentially related to the characteristics of external wind loads over the entire frequency range. The resonant component is regarded as the sum of the inertial and damping force terms. Because the damping of tall buildings is generally low, the effect of damping force term on the resonant component can be neglected. In addition, in the new model, the cross-term between the background and resonant components are formulated. Considering the cross-term, we can estimate the total response more exactly.

Regarding the along-wind responses, the 'Original definition' models provide relatively accurate estimations. When the background component covers the whole frequency range, the overestimation of the responses is less than $5 \%$, which can be acceptable in practical applications. On the other hand, the 'Original definition' models are not suitable for the across-wind responses, because they may underestimate the responses by more than $5 \%$.

We have proposed the new model for estimating the wind-induced responses of tall buildings in the present paper.
This new model can be applied to the other types of buildings: e.g., tall buildings with artificial dampers, soil-structure interaction (SSI) effect or seismic isolation as shown in Fig. 8. If the physical nature of the background and resonant components and their cross-term in the new model is understood well, it will become easier to estimate the equivalent static load effect on these unusual buildings by using this model than the conventional models. In the case of these buildings, there exists the additional acceleration produced by the artificial devices, basement rocking and swaying which can be included into the resonant component based on the new model. On the other hand, because there is significant difference in damping level between the two structural systems of these buildings, the mode shape of vibration may be quite different from that of conventional buildings and difficult to be obtained. Therefore, it may be difficult to estimate the equivalent static load effects based on the 'Original definition' models. By comparison, the new model can be incorporated with the response analysis easily.

\section{Acknowledgement}

This work was partially supported by the scholarship from the Ministry of Education, Science, Sports and Culture of Japan. This support is gratefully acknowledged. The writers are also grateful to Dr. Xinzhong Chen of Texas Tech University, U.S.A., for valuable discussion and suggestions.

\section{References}

1) Davenport, A. G., "The application of statistical concepts to the wind loading of structures." Proceedings, Institution of Civil Engineers, London, Vol. 19, pp. 449-472, (1961)

2) Davenport, A. G., "The response of slender line-like structures to a gusty wind." Proceedings, Institution of Civil Engineers, London, Vol. 23, pp. 389-408, (1962)

3) Davenport, A. G., "Gust loading factors." J. Struct. Div., ASCE, Vol. 93, No. ST3, pp. 11-34, (1967)

4) Repetto, M. P., Solari, G., "Equivalent static wind actions on vertical structures.” J. Wind. Eng. Ind. Aerodyn., Vol. 92, pp. 335-357, (2004)

5) Engineering Sciences Data Unit, "The response of flexible structures to atmospheric turbulence." ESDU76001, London, (1976)

6) Solari, G., "Alongwind response estimation: Closed form solution.” J. Struct. Div., ASCE, Vol. 108, No. ST1, pp. 225-244, (1982) 
7) Kasperski, M., "Extreme wind load distributions for linear and nonlinear design.” Eng. Struct., Vol. 14, pp. 27-34, (1992)

8) Holmes, J. D., "Wind loading of structures." Taylor \& Francis, (2001)

9) Davenport, A. G., "The generalization and simplification of wind loads and implications for computational methods.” J. Wind. Eng. Ind. Aerodyn., Vol. 46-47, pp. 409-417, (1993)

10) Davenport, A. G., "How can we simplify and generalize wind loads?” J. Wind. Eng. Ind. Aerodyn., Vol. 54-55, pp. 657-669, (1995)

11) Holmes, J. D., "Effective static load distributions in wind engineering.” J. Wind. Eng. Ind. Aerodyn., Vol. 90, pp. 91-109, (2002)

12) Kareem, A., Zhou. Y., "Gust loading factor: Past, Present, and Future.” J. Wind. Eng. Ind. Aerodyn., Vol. 91, No. 12-15, pp. 1301-1328, (2003)

13) Chen, X., Kareem, A., "Equivalent static wind loads on tall buildings: New model.” J. Struct. Eng., ASCE, Vol. 130, No. 10, pp. 1425-1435, (2004)

14) Huang, G., Chen, X., "Wind load effects and equivalent static wind loads of tall buildings based on synchronous pressure measurements." Eng. Struct., Vol. 29, pp. 2641-2653, (2007)

15) Vickery, B. J., "The response of chimneys and tower-like structures to wind loading." A State of the Art in Wind Engineering, Wiley Eastern, (1995)

16) Chen, $X .$, Personal communication, (2009)

17) Ashraf Ali, M., Gould, P. L., "On the resonant component of the response of single degree-of-freedom systems under random loading.” Eng. Struct., Vol. 7, No. 4, pp. 280-282, (1985)

18) Chopra, A. K., "Dynamic of structures: Theory and application to earthquake engineering." the $2^{\text {nd }}$ ed., Prentice-Hall, (2000)

19) Newland, D. E., "An introduction to random vibrations, spectral and wavelet analysis." Longman Scientific \& Technical, (1993)

20) $\mathrm{Xu}, \mathrm{Y}$. L., Kwok, K.C.S., "Mode shape corrections for wind tunnel tests of tall buildings.” Eng. Struct., Vol. 15, No. 5, pp. 387-392, (1993)

21) Architectural Institute of Japan, Recommendations for Loads on Buildings, (2004)

\section{Appendix}

1 For along-wind response

(1) Low correlation level:

$$
\frac{\sigma_{\text {Ori. }}}{\sigma_{\text {New }}}=\left\{\frac{\frac{1}{1+2 \alpha+2 \beta_{0}} \int_{0}^{f_{1}-\Delta f^{-}} S_{u}^{*}(f) d f+\frac{(2 \beta+1)^{2}}{(1+2 v+2 \beta)\left(\beta+\beta_{0}+1\right)^{2}} \frac{\pi}{4 \zeta_{1}} f_{1} S_{u}^{*}\left(f_{1}\right)}{\frac{1}{1+2 \alpha+2 \beta_{0}} \int_{0}^{+\infty} S_{u}^{*}(f) d f+\frac{(2 \beta+1)}{(1+2 \alpha+2 \beta)\left(\beta+\beta_{0}+1\right)^{2}} \int_{0}^{+\infty}\left|H_{1}(f)-1\right|^{2} S_{u}^{*}(f) d f+\frac{(2 \beta)}{\left(1+2 \alpha+\beta_{0}+\beta\right)\left(\beta+\beta_{0}+1\right)} \int_{0}^{+\infty}\left\langle H_{1}(f)+H_{1}^{\prime}(f)-2\right\rangle S_{u}^{*}(f) d f}\right\}^{1 / 2}
$$

(2) High correlation level:

$$
\frac{\sigma_{\text {Ori. }}}{\sigma_{\text {New }}}=\left\{\frac{\left(\frac{1}{1+\alpha+\beta_{0}}\right)^{2} \int_{0}^{f_{1}-\Delta f^{-}} S_{u}^{*}(f) d f+\left[\frac{(2 \beta+1)}{(1+\alpha+\beta)\left(\beta+\beta_{0}+1\right)}\right]^{2} \frac{\pi}{4 \zeta_{1}} f_{1} S_{u}^{*}\left(f_{1}\right)}{\left(\frac{1}{1+\alpha+\beta_{0}}\right)^{2} \int_{0}^{+\infty} S_{u}^{*}(f) d f+\left[\frac{(2 \beta+1)}{(1+\alpha+\beta)\left(\beta+\beta_{0}+1\right)}\right]_{0}^{+\infty}\left|H_{1}(f)-1\right|^{2} S_{u}^{*}(f) d f+\frac{(2 \beta+1)}{\left(1+\alpha+\beta_{0}\right)(1+\alpha+\beta)\left(\beta+\beta_{0}+1\right)} \int_{0}^{+\infty}\left\langle H_{1}(f)+H_{1}^{\prime}(f)-2\right\rangle S_{u}^{*}(f) d f}\right\}^{1 / 2}
$$

2 For across-wind response (load effect: base moment)

$$
\begin{aligned}
& \frac{\sigma_{\text {Ori. }}}{\sigma_{\text {New }}}=\left\{\frac{\int_{0}^{f_{1}-\Delta f^{-}} S_{M}(f) d f+\frac{\eta^{2}(2 \beta+1)^{2}}{(\beta+2)^{2}} \frac{\pi}{4 \zeta_{1}} f_{1} S_{M}\left(f_{1}\right)}{\int_{0}^{\infty} S_{M}(f) d f+\frac{\eta^{2}(2 \beta+1)^{2}}{(\beta+2)^{2}} \int_{0}^{+\infty}\left|H_{1}(f)-1\right|^{2} S_{M}(f) d f+\frac{\eta(2 \beta+1)}{(\beta+2)} \int_{0}^{+\infty}\left\langle H_{1}(f)+H_{1}^{\prime}(f)-2\right\rangle S_{M}(f) d f}\right\}^{1 / 2} \\
& \eta= \begin{cases}\frac{2 \alpha+2}{2 \alpha+\beta+1} & \beta \leq 1 \\
\left(\frac{4 \alpha+3}{4 \alpha+2 \beta+1}\right)^{1 / 2} & \beta>1\end{cases}
\end{aligned}
$$

